\title{
POEMS syndrome with vascular transformation of the lymph node sinuses: A case report
}

\author{
XIAOQING WANG ${ }^{1 *}$, XIAOWEN YU $^{2 *}$, DESHENG ZHU $^{1}$, SHEQING ZHANG ${ }^{1}$, \\ XIAJUN ZHOU ${ }^{1}$, MINGYUAN LIU ${ }^{1}$ and YANGTAI GUAN ${ }^{1,3}$ \\ Departments of ${ }^{1}$ Neurology and ${ }^{2}$ Gerontology, Changhai Hospital, Second Military Medical University, Shanghai 200433; \\ ${ }^{3}$ Department of Neurology, Renji Hospital, Shanghai Jiaotong University, Shanghai 200433, P.R. China
}

Received September 25, 2014; Accepted May 14, 2015

DOI: $10.3892 / \mathrm{ol} .2015 .3413$

\begin{abstract}
POEMS syndrome is a rare multisystem disorder associated with the clinical signs of polyneuropathy, organomegaly, endocrinopathy, monoclonal gammopathy and skin changes. However, there is often a delay in the diagnosis due to a lack of overall consideration of the symptoms collectively. For this reason, POEMS syndrome is frequently mistaken for other diseases, such as chronic inflammatory demyelinating polyneuropathy. The present study reports the case of a 40-year-old female patient, who presented with a progressive lack of strength in the lower limbs and a unilateral cervical lump. The patient's enlarged cervical lymph nodes were mistaken for local hemangioma. However, subsequently POEMS syndrome with vascular transformation of the lymph node sinuses (VTS) was diagnosed. The patient received glucocorticoid treatment (20 mg prednisone acetate, daily), which is ongoing. The most recent follow-up examination revealed that the patient's strength had improved and at the time of writing the patient remained alive. The study discusses the clinical manifestations, auxiliary examinations and reason for the misdiagnosis. Hematoxylin and eosin staining and cluster of differentiation 31 immunostaining were adopted to identify the VTS. To the best of our knowledge, this is the first report of POEMS syndrome with VTS.
\end{abstract}

\section{Introduction}

POEMS syndrome, a term coined by Bardwick in 1980 (1), is a rare multisystem disorder associated with the clinical signs of polyneuropathy, organomegaly, endocrinopathy, monoclonal

Correspondence to: Professor Yangtai Guan, Department of Neurology, Changhai Hospital, Second Military Medical University, 1680 Dongfang Road, Shanghai 200433, P.R. China

E-mail: yangtaiguan@sina.com

${ }^{*}$ Contributed equally

Key words: POEMS syndrome, vascular transformation of the lymph node sinuses gammopathy and skin changes (2). However, there is often a delay in diagnosis due to a lack of overall consideration of the symptoms (3). Early diagnosis is critical for POEMS patients to increase survival and reduce the morbidity rate. However, POEMS syndrome is frequently mistaken for other diseases due to its rarity and complex multisystemic manifestations, such as chronic inflammatory demyelinating polyneuropathy (CIDP); it has been reported that $\sim 60 \%$ of patients were misdiagnosed as CIDP (4). POEMS treatment is similar to that administered for patients with suspected myeloma. Radiation therapy is administered for isolated plasma cell tumors. At present, no placebo-controlled studies investigating POEM treatments have been performed, however, systemic treatment may be effective. Currently, the main treatments for POEMS syndrome are limited to the administration of alkylating agents, such as cyclophosphamide, in combination with peripheral blood stem cell transplantation or lenalidomide combined with dexamethasone (4). The estimated median survival time of patients with POEMS syndrome is 14 years (4).

Vascular transformation of the lymph node sinuses (VTS) is a histopathological entity first described by Haferkamp et al (5) in 1971. Obstruction of the efferent lymph node vessel or lymph node venous flow is believed to play an important pathogenetic role in the development of VTS (6). The present study reports the case of a patient with enlarged cervical lymph nodes, initially misdiagnosed as local hemangioma. The clinical manifestations, diagnostic procedure, auxiliary examinations and reason for misdiagnosis are also discussed.

\section{Case report}

On July 29th, 2013, a 40-year-old female presented to Tianshi Shangke Hospital (Lishui, China) with a cervical lump, which was soft without tenderness. An enhanced computed tomography scan of the neck revealed multiple enlarged venous blood vessel masses in the right side of supraclavicular area and upper mediastinum. On September 28th, 2013, the patient was admitted to Changhai Hospital (Shanghai, China) with progressive lack of strength in the lower limbs and a unilateral cervical lump which had been apparent for 8 months. Prior to admission to Changhai Hospital, the patient had experienced thigh numbness. However, as walking, urination, squatting and standing without assistance were unaffected, no special 
Table I. Symptoms relevant to POEMS in the present patient.

\begin{tabular}{ll}
\hline Symptom & \multicolumn{1}{c}{ Evidence } \\
\hline $\begin{array}{l}\text { Polyneuropathy } \\
\text { Organomegaly }\end{array}$ & Progressive lack of strength and numbness in the lower limbs \\
Endocrinopathy & Lymph node enlargement \\
& $\begin{array}{l}\text { Pitting edema in the ankles } \\
\text { and adrenocorticotropic hormone level of } 75.78 \mathrm{pmol} / \mathrm{ml}(\text { normal range, }, 1.39-24.20 \mu \mathrm{g} / \mathrm{l})\end{array}$ \\
Monoclonal gammopathy & Bone marrow puncture revealed a predominance of lymphocytes \\
Skin changes & Skin color of the patient deepened as the disease progressed
\end{tabular}

${ }^{a}$ This symptom was potentially induced by vascular endothelial growth factor (VEGF), which is known to target endothelial cells and induce a reversible and rapid increase in vascular permeability. Therefore, a high level of VEGF can lead to edema.

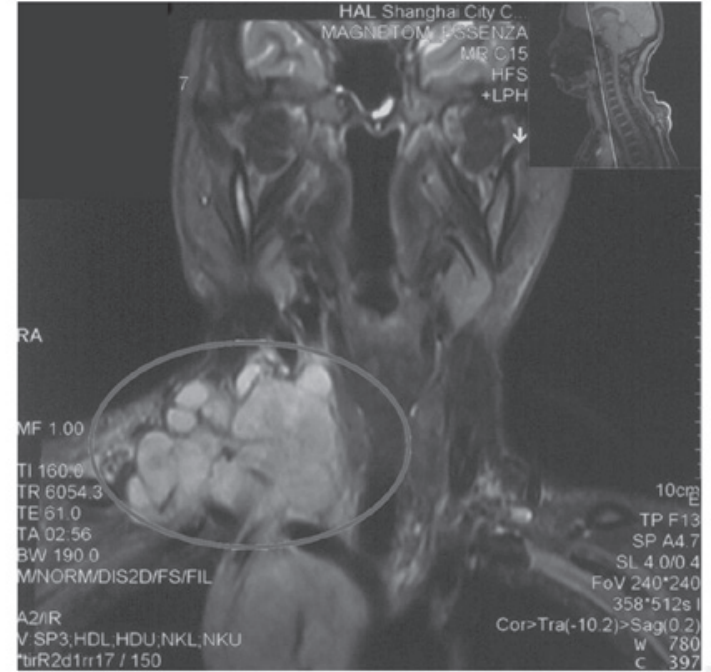

Figure 1. Enhanced magnetic resonance imaging scan showing a unilateral lesion in the cervical region (circle).

treatment had been offered. The symptoms persisted and subsequently progressed; 4 months after the onset of thigh numbness, the patient could no longer walk unaided. No headaches, photophobia, incontinence or coughing were reported throughout. The patient's medical history included no underlying health problems. Upon neurophysical examination on September 28th, 2013, motor strength and deep tendon reflexes were weak. Motor strength was rated at grade 4 (according to the Medical Research Council's Scale for Muscle Strength) (7) in the lower extremities, and soft pitting edema was observed in the ankles. Atrophy of the calf and thenar muscles and steppage gait were also present. On September 30th, 2013, possible metastases with a rich blood supply were observed on an enhanced magnetic resonance imaging scan of the region (Fig. 1). However, an ultrasound scan performed on the same day showed enlargement of the cervical lymph nodes. Subsequently, local hemangioma was misdiagnosed. On October 11th, 2013, a lumbar puncture was performed for a presumed diagnosis of peripheral neuropathy. Cerebral spinal fluid analysis showed an elevated protein level of $1.68 \mathrm{~g} / \mathrm{l}$ (normal range, $0.15-0.45 \mathrm{~g} / \mathrm{l}$ ), a normal white blood cell count of $1 / \mu 1$ (normal, $\leq 5 / \mu 1$ ), no malignant cells and a normal glucose level. A suspected diagnosis of CIDP was concluded. However, on October 12th, 2013, the patient's husband reported that the patient's skin color had become deeper as the disease had progressed.

Consequently, a series of auxiliary examinations were performed on the same day, concentrating on symptoms associated with POEMS syndrome, was performed. Table I shows the POEMS syndrome-related symptoms identified in the present patient. This included a predominance of lymphocytes observed on a bone marrow smear (Fig. 2).

To clarify the characteristics of the mass, a biopsy of the superficial lymph node was performed on October 23rd, 2013 (Fig. 3A). This was diagnosed as VTS (Fig. 3B and C) by hematoxylin and eosin staining and CD31 immunostaining as CD31 staining was observed in the blood vessels of the lymph node sinuses (Fig. 3D and E). However, the biopsy result did not account for all the symptoms that the patient possessed, such as the weakness in the lower limbs. The patient was administered glucocorticoids (20 mg prednisone acetate, daily). At the most recent follow-up examination, in December 2014, a slight improvement in the patient's symptoms was observed and at the time of writing the patient remained alive.

\section{Discussion}

POEMS syndrome is a rare condition characterized by polyneuropathy, organomegaly, endocrinopathy, monoclonal gammopathy and skin lesions (8). The diagnosis is based on a combination of clinical and laboratory features, and misdiagnosis may occur if the symptoms are not considered collectively. In the present study, the patient's clinical characteristics supported the diagnosis of POEMS syndrome based on the evidence described in Table I. It is often challenging to correctly diagnose this condition initially, as all of the typical features may not appear concurrently.

The mechanism of POEMS syndrome remains unclear (9), however, it has been reported that overproduction of vascular endothelial growth factor (VEGF) may be of great importance in the pathogenesis of polyneuropathy, due to the induction of angiogenesis. It has also been demonstrated that VEGF levels are higher in patients with POEMS syndrome compared with normal controls (10). Theoretically, these angiogenic factors may enter the regional lymph nodes and induce the changes associated with VTS. 

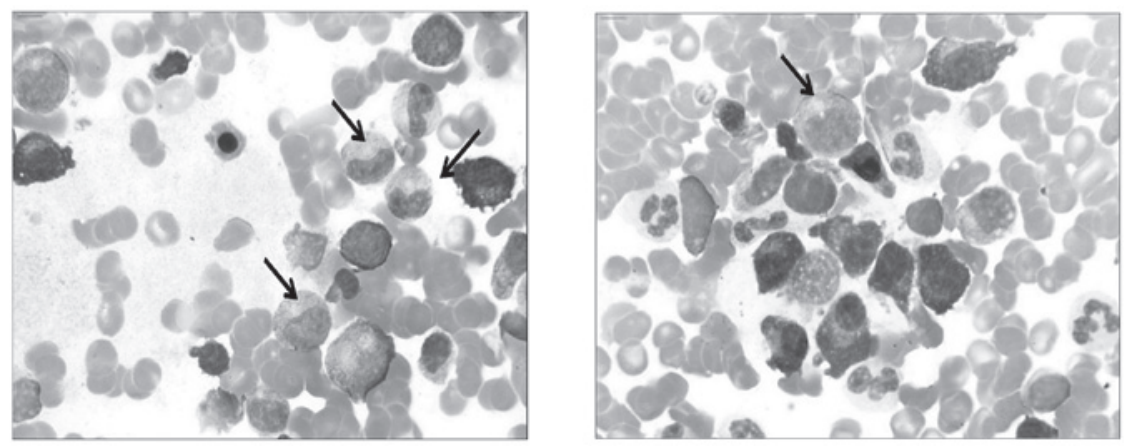

Figure 2. Bone marrow smear; arrows indicate the increased plasma cells (magnification, x100).

A
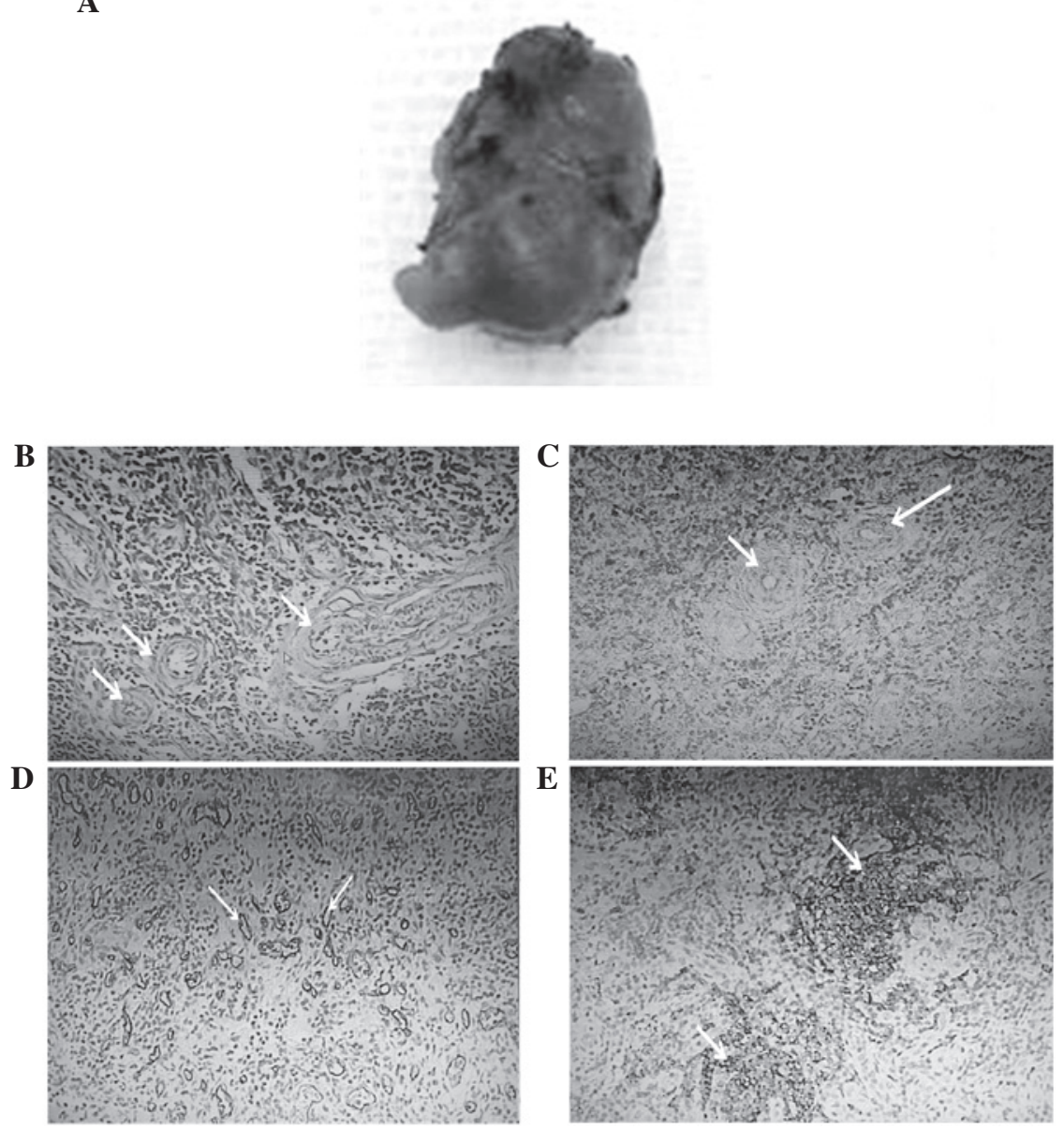

Figure 3. (A) Biopsy specimen of the diseased cervical lymphoid tissue, $0.5 \times 1.1 \times 2.1 \mathrm{~cm}$ in size. (B and C) Vascular transformations of the lymph node sinuses (arrows; hematoxylin and eosin stain; magnification, B, x40 and C, x10). (D and E) Cluster of differentiation 31 immunostaining of the vascular transformation of the lymph node sinuses (arrows) (magnification, D, x4 and E, x40).

VTS is a benign lymph node vascular hyperplastic lesion; although it is not a true tumor, it may be confused with cancerous tumors or metastases. VTS is considered to occur secondary to obstruction of the lymph node venous or efferent vessels $(11,12)$. In the present case, abnormal clonal plasma cell proliferation, characteristic of POEMS, was identified in the bone marrow, suggesting a potentially severe outflow problem in the lymph circulation.

From a diagnostic perspective, a lump in the neck region may not appear to be associated with POEMS syndrome, a systemic disease; therefore, the monistic nature of this case was not initially apparent. When the case was considered as a whole, together with the local aspects, in the current patient, it was deduced that VTS of the bilateral cervical region was a regional lesion induced by the pathological changes associated with POEMS syndrome.

In conclusion, the current study presents a rare association of cervical lymph node enlargement due to VTS in a patient with POEMS syndrome which, to the best of our knowledge, has not previously been reported. This case highlights the 
importance of detecting VEGF expression in patients with POEMS syndrome and we recommend that patients are followed up closely.

\section{Acknowledgements}

This study was supported by grants from the National Natural Science Foundations of China (nos. 81200924 and 81230027), the Natural Science Foundations of Shanghai (no. 12ZR1427400), the Outstanding Subject Leaders Project of Shanghai (no. 14XD1403400) and the Technology Support Project of Shanghai (no. 14140903300).

\section{References}

1. Bardwick PA, Zvaifler NJ, Gill GN, Newman D, Greenway GD and Resnick DL: Plasma cell dyscrasia with polyneuropathy, organomegaly, endocrinopathy, $\mathrm{M}$ protein, and skin changes: The POEMS syndrome. Report on two cases and a review of the literature. Medicine (Baltimore) 59: 311-322, 1980

2. Piradov MA, Suponeva NA, Ginzberg MA, Nikitin SS Varlamova EIu, Ryzhko VV, Semochkin SV and Merkulova DM: POEMS-syndrome: A literature review and case reports. Zh Nevrol Psikhiatr Im S S Korsakova 114: 4-10, 2014 (In Russian).

3. Briani C, Dalla Torre C, Lessi F, Cavallaro T, Scarlato M, Ferrari S, Campagnolo M, Lucchetta M, Cabrini I, Morbin M, Lauria G, Adami $F$ and Manfredi AA: Pentraxin-3 and VEGF in POEMS syndrome: A 2-year longitudinal study. J Neuroimmunol 277: 189-192, 2014.
4. Latov N: Diagnosis and treatment of chronic acquired demyelinating polyneuropathies. Nat Rev Neurol 10: 435-446, 2014.

5. Haferkamp O, Rosenau W and Lennert K: Vascular transformation of lymph node sinuses due to venous obstruction. Arch Pathol 92: 81-83, 1971.

6. Ostrowski ML, Siddiqui T, Barnes RE and Howton MJ: Vascular transformation of lymph node sinuses. A process displaying a spectrum of histologic features. Arch Pathol Lab Med 114: 656-660, 1990.

7. Kim GW, Won YH, Park SH, Seo JH and Ko MH: Can motor evoked potentials be an objective parameter to assess extremity function at the acute or subacute stroke stage? Ann Rehabil Med 39: 253-261, 2015.

8. Li J and Zhou DB: New advances in the diagnosis and treatment of POEMS syndrome. Br J Haematol 161: 303-315, 2013.

9. Zhang X, Chen K, Shi S, Liu Z, Jin Y and Hu J: A rare case of POEMS syndrome initially presenting with trigeminal neuralgia. Clin Neurol Neurosurg 115: 89-90, 2013.

10. Dispenzieri A: POEMS syndrome: 2014 update on diagnosis, risk-stratification, and management. Am J Hematol 89: 214-223, 2014

11. Meysman M, Diltoer M, Raeve HD, Monsieur I and Huyghens L: Chronic thromboembolic pulmonary hypertension and vascular transformation of the lymph node sinuses. Eur Resp J 10: 1191-1193, 1997.

12. Pirola S, Shenjere P and Nonaka D: Combined usual and nodular types of vascular transformation of sinuses in the same lymph node. Int J Surg Pathol 20: 175-177, 2012. 